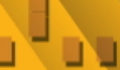

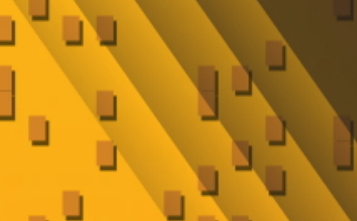

$$
\begin{aligned}
& \text { AVgebra \& } \\
& \text { Number } \\
& \text { Theory } \\
& \text { Dolume } 4
\end{aligned}
$$

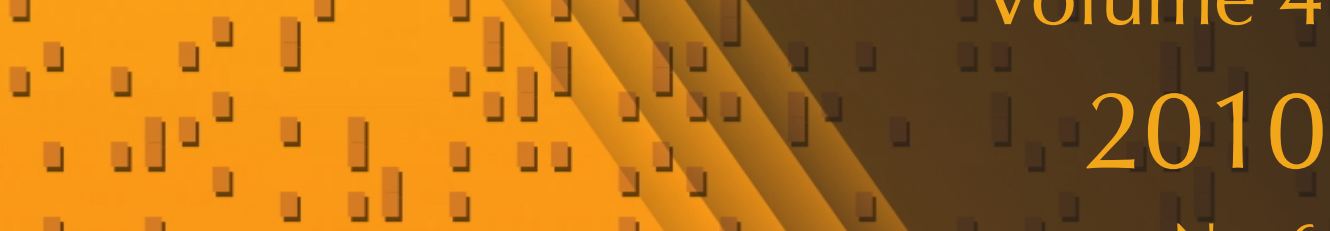

$$
\begin{aligned}
& \text { 」ป } \\
& \text { 」Ј }
\end{aligned}
$$

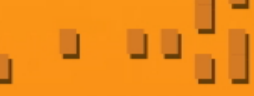

$$
\begin{aligned}
& \text { ป」」」」」」 } \\
& \lrcorner\lrcorner \\
& \text { No. } 6 \\
& \lrcorner
\end{aligned}
$$

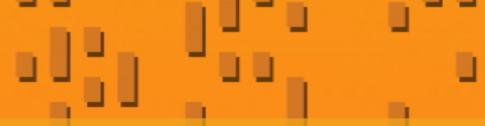

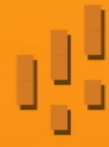

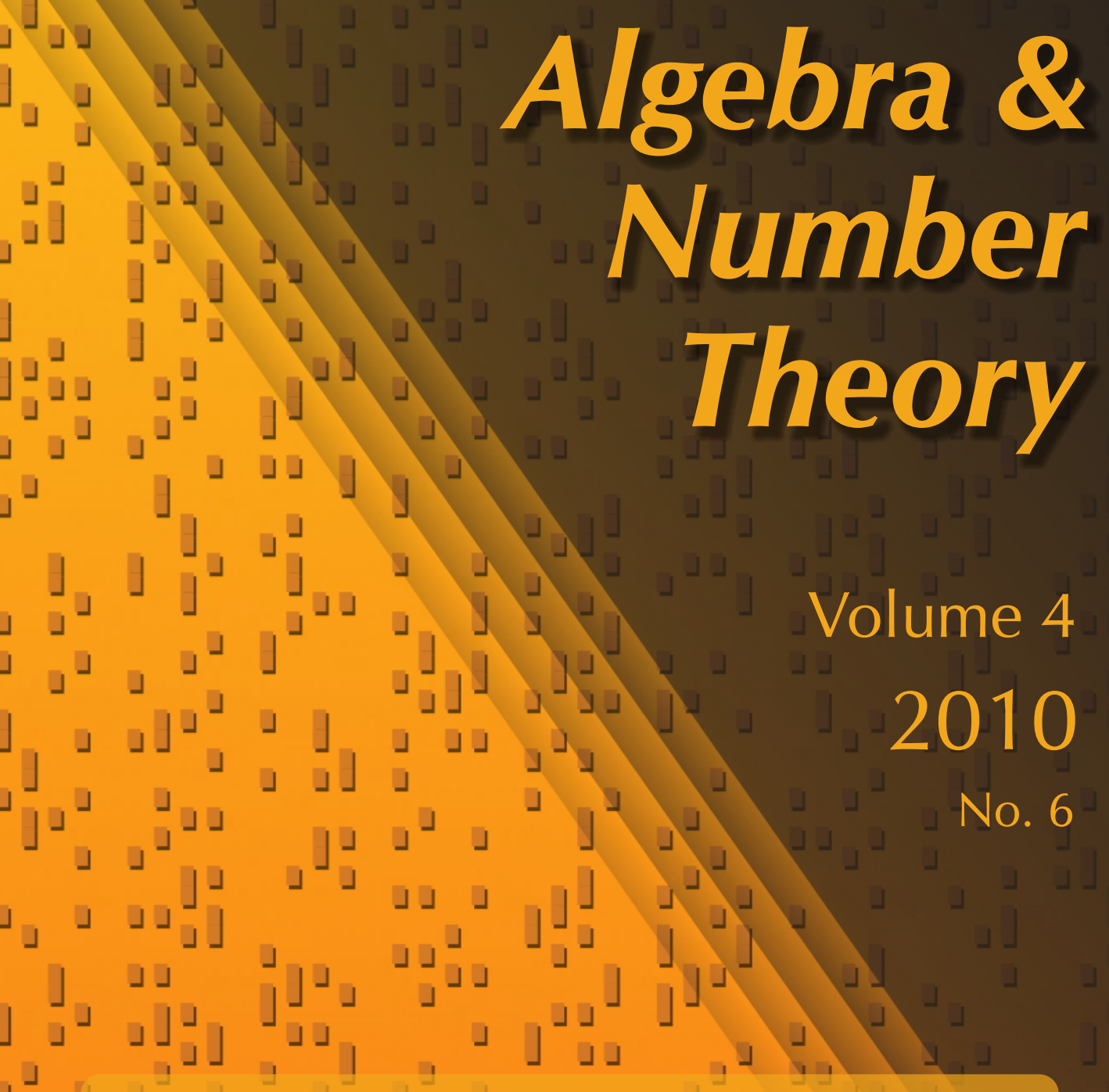

Patching and admissibility over two-dimensional complete local domains

Danny Neftin and Elad Paran

」

4 Dany Neftin and Elad Paran

$\neg$




\title{
Patching and admissibility over two-dimensional complete local domains
}

\author{
Danny Neftin and Elad Paran
}

\begin{abstract}
We develop a patching machinery over the field $E=K((X, Y))$ of formal power series in two variables over an infinite field $K$. We apply this machinery to prove that if $K$ is separably closed and $G$ is a finite group of order not divisible by char $E$, then there exists a $G$-crossed product algebra with center $E$ if and only if the Sylow subgroups of $G$ are abelian of rank at most 2 .
\end{abstract}

\section{Introduction}

Complete local domains play an important role in commutative algebra and algebraic geometry, and their algebraic properties were already described in 1946 by Cohen's structure theorem. The Galois theoretic properties of their quotient fields were extensively studied over the past two decades. The pioneering work in this line of research is due to Harbater [1987], who introduced the method of patching to prove that if $R$ is a complete local domain with quotient field $K$, then every finite group occurs as a Galois group over $K(x)$. This result was strengthened by Pop [1996] and, in a different language, by Haran and Jarden [1998], who showed that if moreover $R$ is of dimension 1, then every finite split embedding problem over $K(x)$ is solvable.

The first step towards higher dimension was made by Harbater and Stevenson [2005], who essentially showed that if $R$ is a complete local domain of dimension 2 , then every finite split embedding problem over Quot $R$ has $|R|$ independent solutions. That is, the absolute Galois group of Quot $R$ is semifree of rank $|R|$ (see [Bary-Soroker et al. 2008] for details on this notion). This result was generalized in [Pop 2010; Paran 2010], where it is shown that if $K$ is the quotient field of a complete local domain of dimension exceeding 1 , then $\mathrm{Gal} K$ is semifree.

Despite the major progress made in the study of Galois theory over these fields, little is known about the structure of division algebras over them. A step in that

MSC2000: primary 12E30; secondary $16 \mathrm{~S} 35$.

Keywords: patching, admissible groups, division algebras, complete local domains.

The second author was partially supported by the Israel Science Foundation (grant No. 343/07), and by an ERC grant. 
direction was recently made by Harbater et al. [2009], who consider a question relating Galois theory and Brauer theory over a field $E$ : Which groups are admissible over $E$ ? That is, which finite groups occur as a Galois group of an adequate Galois extension $F / E$ (recall that an extension $F / E$ is called adequate if $F$ is a maximal subfield in an $E$-central division algebra). Equivalently, for which groups $G$ there is a $G$-crossed product division algebra with center $E$. Note that for $E$ as above and a finite extension $F / E$, the above maximality requirement can be omitted since any $F$ which is a subfield of an $E$-division algebra is also a maximal subfield of some $E$-division algebra (see Remark 3.9).

This question was first considered by Schacher [1968] over global fields. He proved that any $\mathbb{Q}$-admissible group has metacyclic Sylow subgroups and conjectured the converse. Admissibility has been studied extensively over global fields [Stern 1982; Sonn 1983; Schacher and Sonn 1992; Liedahl 1996; Feit 2004; Neftin 2009], function fields, and fields of Laurent series [Fein et al. 1992; Fein and Schacher 1995a; 1995b].

The main theorem of [Harbater et al. 2009] asserts that if $E$ is a function field in one variable over a complete discretely valued field with an algebraically closed residue field, then a finite group $G$ of order not divisible by char $E$ is admissible over $E$ if and only if each of the Sylow subgroups of $G$ is abelian of rank at most 2 (i.e., generated by two elements).

In this work, we take the next natural step, and determine the admissible groups over quotient fields of equicharacteristic (that is, having the same characteristic as their residue field) two-dimensional complete local domains, with a separably closed residue field. In particular, we determine the admissible groups over $C((X, Y))$, whenever $C$ is a separably closed field. This problem was posed to the first author by David Harbater. We show that the result of [Harbater et al. 2009] holds over these fields as well.

Main Theorem. Let $R$ be an equicharacteristic complete local domain of dimension 2, with a separably closed residue field. Let $G$ be a finite group of order not divisible by char $R$. Then $G$ is admissible over Quot $R$ if and only if each of its Sylow subgroups is abelian of rank at most 2.

The "only if" part of this result is essentially proven in [Harbater et al. 2009], using results of [Colliot-Thélène et al. 2002].

The "if" part actually holds in greater generality — the residue field need not be separably closed, it suffices that it contains a primitive root of unity of order $k$, for each $k \in \mathbb{N}$ not divisible by char $R$ (Proposition 3.7). Our proof strategy for the backward direction is as follows. We first use Cohen's structure theorem to reduce the problem from Quot $R$ to a field $E$ of the form $K((X, Y))$. We then apply a patching argument as in [Harbater et al. 2009]; we explicitly realize each 
Sylow subgroup of $G$ by a Galois extension of $E$ which is a maximal subfield in some $E$-central division algebra. We then patch these realizations into a Galois extension $F / E$ with group $G$, in a way that also patches the division algebras into an $E$-central division algebra $D$ containing $F$ as a maximal subfield.

A key ingredient in our proof is a patching machinery over fields of the form $K((X, Y))$, where $K$ may be an arbitrary infinite field. In [Pop 2010; Paran 2010], problems over $K((X, Y))$ are lifted to $K((X, Y))(Z)$, solved there (via different methods), and then the solutions are specialized into solutions over $K((X, Y))$ using Hilbertianity. This approach seems inapplicable to our current goal, since adequate extensions usually do not remain adequate under specialization. Instead we patch groups directly over $K((X, Y))$. To this end we define "analytic fields" over $K((X, Y))$, satisfying the axioms of algebraic patching (i.e., matrix factorization and intersection), as presented in [Haran and Jarden 1998] (a slightly different axiomatization from the "field patching" axiomatization of [Harbater and Hartmann 2007]). The construction of these analytic rings is of a rigid-geometric nature. In recent communication with David Harbater, we learned that a formal-geometric analogue of this form of patching was carried out by him in [2003, Theorem 5.3.9] in order to solve split embedding problems over the field $\mathbb{C}((X, Y))$ of formal power series in two variables over the complex numbers. The core patching arguments in the proof of [Harbater 2003, Theorem 5.3.9] can be extended to replace $\mathbb{C}$ by an arbitrary field and used to study admissible groups, in a similar fashion to our development here.

\section{Analytic fields}

In this section we establish our patching machinery. Fix an infinite field $K$, and let $E=K((X, Y))=\operatorname{Quot}(K \llbracket X, Y \rrbracket)$ be the field of formal power series over $K$ in the variables $X$ and $Y$. Denote by $v$ the order function of the maximal ideal $\langle X, Y\rangle$ in $K \llbracket X, Y \rrbracket$. Then $v$ extends uniquely to a discrete rank-1 valuation of $E$. Note that $K \llbracket X, Y \rrbracket$ is strictly contained in the valuation ring of $v$ in $E$.

Construction 1.1 (analytic rings over $E$ ). Let $I$ be a finite set. For each $i \in I$ let $c_{i} \in K$, such that $c_{i} \neq c_{j}$ for $i \neq j$ (such a choice is possible since $K$ is infinite). For each $i \in I$ denote $z_{i}=Y /\left(X-c_{i} Y\right) \in E$. For each $J \subseteq I$, consider the subring $K\left[z_{j} \mid j \in J\right][X, Y]$ of $E$, and let $D_{J}$ be the completion of this ring with respect to $v$. Note that for each $J \subseteq I, D_{J} \subseteq D_{I}$, and that $D_{\varnothing}=K \llbracket X, Y \rrbracket$, since $K \llbracket X, Y \rrbracket$ is complete. Let $Q=$ Quot $D_{I}$, and for each $i \in I$ let $Q_{i}=E \cdot D_{I \backslash\{i\}}$ and $Q_{i}^{\prime}=\bigcap_{j \neq i} Q_{j}$.

For the rest of this section, we fix the notation of Construction 1.1. A geometric interpretation of the rings defined in Construction 1.1 appears in Remark 1.12. In order to present this interpretation, we need several lemmas. 
Lemma 1.2. Let $i \in I$. Then $v$ is trivial on $K\left(z_{i}\right)$.

Proof. It suffices to prove that $v$ is trivial on $K\left[z_{i}\right]$. Let $0 \neq f=\sum_{n=0}^{d} a_{n} z_{i}^{n} \in K\left[z_{i}\right]$, with $a_{0}, \ldots, a_{d} \in K$. Without loss of generality, $a_{0} \neq 0$. We have

$$
\sum_{n=0}^{d} a_{n} z_{i}^{n}=\frac{\sum_{n=0}^{d} a_{n} Y^{n}\left(X-c_{i} Y\right)^{d-n}}{\left(X-c_{i} Y\right)^{d}} .
$$

By opening parentheses, the numerator in this expression can be written as a sum of monomials of degree $d$. For $n=0$ we get the summand $a_{0} X^{d}$, while all other monomials in this presentation have a positive power of $Y$, so they do not cancel $a_{0} X^{d}$. Thus the numerator has value $d$, and clearly so does the denominator, so $v(f)=0$.

Corollary 1.3. The valuation $v$ is trivial on $K\left[z_{i} \mid i \in I\right]$.

Proof. Note that for each $i, j \in I$ we have $K\left(z_{i}\right)=K\left(z_{j}\right)$. Thus by the preceding lemma, $v$ is trivial on $K\left(z_{i}\right)=K\left(z_{j} \mid j \in I\right)$, and in particular $v$ is trivial on the subring $K\left[z_{i} \mid i \in I\right]$.

Lemma 1.4. Let $J \subseteq I$ and $j \in J$. Then the ring $K\left[z_{l} \mid l \in J\right]\left[X-c_{j} Y\right]$ is isomorphic to the ring of polynomials in one variable over $K\left[z_{l} \mid l \in J\right]$.

Proof. To prove the claim we show that if $\sum_{n=0}^{d} a_{n}\left(X-c_{j} Y\right)^{n}=0$, for $a_{0}, \ldots, a_{d} \in$ $K\left[z_{l} \mid l \in J\right]$, then $a_{0}=\cdots=a_{d}=0$. If not, suppose (without loss of generality) that $a_{0} \neq 0$. By Corollary 1.3, $v\left(a_{0}\right)=0$ while $v\left(a_{k}\left(X-c_{j} Y\right)^{k}\right)=v\left(a_{k}\right)+k=k>0$ for each $k>0$. Hence $\infty=v(0)=v\left(\sum_{n=0}^{d} a_{n}\left(X-c_{j} Y\right)^{n}\right)=0$, a contradiction.

By Lemma 1.4, for each $J \subseteq I, j \in J$, each element of $K\left[z_{l} \mid l \in J\right]\left[X-c_{j} Y\right]$ has a unique presentation as a polynomial in $X-c_{j} Y$. Thus we have a natural valuation on this ring, given by $v^{\prime}\left(\sum_{n=0}^{d} a_{n}\left(X-c_{j} Y\right)^{n}\right)=\min \left(n \mid a_{n} \neq 0\right)$, and we may form the completion $K\left[z_{l} \mid l \in J\right] \llbracket X-c_{j} Y \rrbracket$ of this ring with respect to $v^{\prime}$.

Proposition 1.5. Let $J \subseteq I$ and $j \in J$. Then $D_{J}=K\left[z_{l} \mid l \in J\right] \llbracket X-c_{j} Y \rrbracket$, and $v$ is given on $D_{J}$ by $v\left(\sum_{n=0}^{\infty} a_{n}\left(X-c_{j} Y\right)^{n}\right)=\min \left(n \mid a_{n} \neq 0\right)$.

Proof. By Lemma 1.4, $v$ coincides with $v^{\prime}$ (given in the paragraph preceding this proposition) on $K\left[z_{l} \mid l \in J\right]\left[X-c_{j} Y\right]$; hence $K\left[z_{l} \mid l \in J\right] \llbracket X-c_{j} Y \rrbracket$ is the completion of $K\left[z_{l} \mid l \in J\right]\left[X-c_{j} Y\right]$ with respect to $v$, and $v$ coincides with $v^{\prime}$ on the completion. Note that $K\left[z_{l} \mid l \in J\right]\left[X-c_{j} Y\right]=K\left[z_{l} \mid l \in J\right][X, Y]$ (since $Y=z_{j}\left(X-c_{j} Y\right)$ and $\left.X=\left(1+c_{j} z_{j}\right)\left(X-c_{j} Y\right)\right)$; hence by the definition of $D_{J}$ we are done. 
Lemma 1.6. Let $J \subseteq I$. Then $K\left[z_{j} \mid j \in J\right]=\sum_{j \in J} K\left[z_{j}\right]$.

Proof. For each $i \neq j \in J$ we have

$$
z_{i} \cdot z_{j}=\frac{Y^{2}}{\left(X-c_{i} Y\right) \cdot\left(X-c_{j} Y\right)}=\frac{1}{c_{i}-c_{j}} \cdot z_{i}+\frac{1}{c_{j}-c_{i}} z_{j}
$$

The claim now follows by induction on $|I|$.

Proposition 1.7. Let $J, J^{\prime} \subseteq I$. Then for each $f \in D_{J \cup J^{\prime}}$ there exist $f_{1} \in D_{J}$ and $f_{2} \in D_{J^{\prime}}$ with $v\left(f_{1}\right), v\left(f_{2}\right) \geq v(f)$, such that $f=f_{1}+f_{2}$.

Proof. Replace $J$ with $J \backslash\left(J \cap J^{\prime}\right)$ to assume that $J \cap J^{\prime}=\varnothing$. Moreover, without loss of generality $J, J^{\prime}$ are nonempty. Choose $j \in J$ and $j^{\prime} \in J^{\prime}$, and let

$$
A_{J}=K\left[z_{l} \mid l \in J\right], \quad A_{J^{\prime}}=K\left[z_{l} \mid l \in J^{\prime}\right], \quad A=K\left[z_{l} \mid l \in J \cup J^{\prime}\right] .
$$

By Proposition 1.5, $D_{J}=A_{J} \llbracket X-c_{j} Y \rrbracket, D_{J^{\prime}}=A_{J} \llbracket X-c_{j^{\prime}} Y \rrbracket$, and $D_{J \cup J^{\prime}}=$ $A \llbracket X-c_{j} Y \rrbracket$. Let $f=\sum_{n=m}^{\infty} a_{n}\left(X-c_{j} Y\right)^{n} \in D_{J \cup J^{\prime}}$, with $a_{m} \neq 0$. Then $v(f)=m$ by Proposition 1.5. By Lemma 1.6, $A=A_{J}+A_{J^{\prime}}$. For each $n \geq m$, let $b_{n} \in$ $A_{J}, b_{n}^{\prime} \in A_{J^{\prime}}$ such that $a_{n}=b_{n}+b_{n}^{\prime}$. Let $f_{1}=\sum_{n=m}^{\infty} b_{n}\left(X-c_{j} Y\right)^{n}$ and $f_{2}=$ $f-f_{1}=\sum_{n=m}^{\infty} b_{n}^{\prime}\left(X-c_{j} Y\right)^{n}$. Then $f_{1} \in D_{J}$ and $v\left(f_{1}\right) \geq m$. It remains to prove that $f_{2} \in D_{J^{\prime}}$ and that $v\left(f_{2}\right) \geq m$. This follows by the equality:

$$
\begin{aligned}
f_{2}=\sum_{n=m}^{\infty} b_{n}^{\prime}\left(X-c_{j} Y\right)^{n} & =\sum_{n=m}^{\infty} b_{n}^{\prime}\left(\left(X-c_{j^{\prime}} Y\right)+\left(c_{j^{\prime}}-c_{j}\right) Y\right)^{n} \\
& =\sum_{n=m}^{\infty}\left(b_{n}^{\prime}\left(1+\left(c_{j^{\prime}}-c_{j}\right) z_{j^{\prime}}\right)^{n}\right)\left(X-c_{j^{\prime}} Y\right)^{n} .
\end{aligned}
$$

The next lemma is a variant of [Harbater and Hartmann 2007, Lemma 3.3], allowing nonprincipal ideals.

Lemma 1.8. Let $R \subseteq R_{1}$ and $R_{2} \subseteq R_{0}$ be domains such that $R_{0}=R_{1}+R_{2}$. Let $w$ be a nontrivial discrete valuation on Quot $R_{0}$ such that $R$ is complete with respect to $w$ and $w(x) \geq 0$ for all $x \in R_{0}$. Let $\mathfrak{p}, \mathfrak{p}_{1}, \mathfrak{p}_{2}$, and $\mathfrak{p}_{0}$ be the centers of $w$ in $R$, $R_{1}, R_{2}$, and $R_{0}$, respectively. Suppose that $\mathfrak{p} R_{0}=\mathfrak{p}_{0}$ and $R / \mathfrak{p}=R_{1} / \mathfrak{p}_{1} \cap R_{2} / \mathfrak{p}_{2}$ (inside $R_{0} / \mathfrak{p}_{0}$ ). Then $R_{1} \cap R_{2}=R$.

Proof. First, note that $\mathfrak{p}_{0}=\mathfrak{p}_{1}+\mathfrak{p}_{2}$. Indeed, suppose $x \in \mathfrak{p}_{0}=\mathfrak{p} R_{0}$. Then $x=$ $\sum_{i=1}^{n} a_{i} x_{i}$ for some $a_{1}, \ldots, a_{n} \in R_{0}$ and $x_{1}, \ldots, x_{n} \in \mathfrak{p}$. For each $1 \leq i \leq n$, write $a_{i}=b_{i}+b_{i}^{\prime}$ with $b_{i} \in R_{1}$ and $b_{i}^{\prime} \in R_{2}$. Then $\sum a_{i} x_{i}=\sum b_{i} x_{i}+\sum b_{i}^{\prime} x_{i} \in \mathfrak{p}_{1}+\mathfrak{p}_{2}$, since $\mathfrak{p} \subseteq \mathfrak{p}_{1}, \mathfrak{p}_{2}$.

Let $S=R_{1} \cap R_{2}$ and $\mathfrak{q}$ be the center of $w$ at $S$. Then the sequence $0 \rightarrow S \rightarrow$ $R_{1} \times R_{2} \rightarrow R_{0} \rightarrow 0$ is exact (where the second map is the diagonal map and the third map is substraction). This sequence induces an exact sequence $0 \rightarrow S / \mathfrak{q} \rightarrow$ $\left(R_{1} / \mathfrak{p}_{1}\right) \times\left(R_{2} / \mathfrak{p}_{2}\right) \rightarrow R_{0} / \mathfrak{p}_{0} \rightarrow 0$. Indeed, the only nontrivial part in showing this 
is to check that the kernel of the substraction map is contained in the image of the diagonal map. Suppose $\left(x_{1}+\mathfrak{p}_{1}, x_{2}+\mathfrak{p}_{2}\right) \in\left(R_{1} / \mathfrak{p}_{1}\right) \times\left(R_{2} / \mathfrak{p}_{2}\right)$ satisfies $x_{1}-x_{2} \in \mathfrak{p}_{0}$. Since $\mathfrak{p}_{0}=\mathfrak{p}_{1}+\mathfrak{p}_{2}$ we may choose $y_{1} \in \mathfrak{p}_{1}, y_{2} \in \mathfrak{p}_{2}$ such that $x_{1}-y_{1}=x_{2}-y_{2}$. Then $\left(x_{1}+\mathfrak{p}_{1}, x_{2}+\mathfrak{p}_{2}\right)=\left(x_{1}-y_{1}+\mathfrak{p}_{1}, x_{2}-y_{2}+\mathfrak{p}_{2}\right)$ belongs to the image of the diagonal map. Thus the sequence is exact.

Since $\mathfrak{p}$ is the center of $w$ on $R$ and $\mathfrak{p}_{1}$ the center of $w$ on $R_{1}$, we have $R \cap \mathfrak{p}_{1}=\mathfrak{p}$. In particular, the diagonal map $R / \mathfrak{p} \rightarrow\left(R_{1} / \mathfrak{p}_{1}\right) \times\left(R_{2} / \mathfrak{p}_{2}\right)$ is injective. Since $R_{0}=$ $R_{1}+R_{2}$, the substraction map $\left(R_{1} / \mathfrak{p}_{1}\right) \times\left(R_{2} / \mathfrak{p}_{2}\right) \rightarrow R_{0} / \mathfrak{p}_{0}$ is surjective. Thus, since $R / \mathfrak{p}=R_{1} / \mathfrak{p}_{1} \cap R_{2} / \mathfrak{p}_{2}$, the sequence $0 \rightarrow R / \mathfrak{p} \rightarrow\left(R_{1} / \mathfrak{p}_{1}\right) \times\left(R_{2} / \mathfrak{p}_{2}\right) \rightarrow R_{0} / \mathfrak{p}_{0} \rightarrow 0$ is also exact. It follows that the natural map $R / \mathfrak{p} \rightarrow S / \mathfrak{q}$ is an isomorphism. In particular, $S=R+\mathfrak{p} S$. By induction we have $S=R+\mathfrak{p}^{k} S$ for each $k \in \mathbb{N}$. Since $\mathfrak{p}_{0}=\mathfrak{p} R_{0}, \mathfrak{p} \neq 0$. Since $w$ is discrete, there exists an integer $m$ such that $v(x) \geq m$ for each $x \in \mathfrak{p}$. Thus $v(x) \geq m k$ for each $x \in \mathfrak{p}^{k}$; hence $R$ is $w$-dense in $S$ and therefore the completion of $R$ with respect to $w$ contains $S$. By our assumptions, $R$ is complete; hence $R=S$.

Lemma 1.9. The set $\left\{z_{i}^{n} \mid i \in I, n \in \mathbb{N} \cup\{0\}\right\}$ is K-linearly independent.

Proof. Suppose $a_{0}+\sum_{i \in I} \sum_{n=1}^{d_{i}} a_{i, n} z_{i}^{n}=0$, where $d_{i} \in \mathbb{N}$ and $a_{0}, a_{i, n} \in K$ for each $i$ and $n$. We wish to show that $a_{0}=a_{i, n}=0$ for each $i$ and $n$. Suppose there exist $i \in I$ and $n \in \mathbb{N}$ such that $a_{i, n} \neq 0$. Without loss of generality, $n=d_{i}$. Since $X-c_{i} Y$ is a prime element of $K[X, Y]$, it defines a discrete valuation on $K(X, Y)$, which we denote by $w$. We have $w(Y)=w\left(Y-c_{j} X\right)=0$ for each $j \neq i$ in $I$. Thus $w\left(a_{0}+\sum_{j \neq i} \sum_{n=1}^{d_{j}} a_{j, n} z_{j}^{n}\right) \geq 0$, while $w\left(\sum_{n=1}^{d_{i}} a_{i, n} z_{i}^{n}\right)=-d_{i}$. Thus $w(0)=w\left(a_{0}+\sum_{j \in I} \sum_{n=1}^{d_{j}} a_{j, n} z_{j}^{n}\right)=-d_{i}$, a contradiction.

Proposition 1.10. Suppose $J, J^{\prime} \subseteq I$. Then $D_{J} \cap D_{J^{\prime}}=D_{J \cap J^{\prime}}$.

Proof. Clearly, $D_{J \cap J^{\prime}} \subseteq D_{J} \cap D_{J^{\prime}}$. For the converse inclusion, we distinguish between two cases. First suppose that $J \cap J^{\prime} \neq \varnothing$, and fix $j \in J \cap J^{\prime}$. Then $D_{J}=K\left[z_{k} \mid k \in J\right] \llbracket X-c_{j} Y \rrbracket$ and $D_{J^{\prime}}=K\left[z_{k} \mid k \in J^{\prime}\right] \llbracket X-c_{j} Y \rrbracket$; hence $D_{J} \cap D_{J^{\prime}}=\left(K\left[z_{k} \mid k \in J\right] \cap K\left[z_{k} \mid k \in J^{\prime}\right]\right) \llbracket X-c_{j} Y \rrbracket$. By Lemma $1.9 K\left[z_{k} \mid k \in J\right]$ $\cap K\left[z_{k} \mid k \in J^{\prime}\right]=K\left[z_{k} \mid k \in J \cap J^{\prime}\right]$; hence $y \in D_{J \cap J^{\prime}}$.

Now suppose that $J \cap J^{\prime}=\varnothing$ and let $R=K \llbracket X, Y \rrbracket=D_{\varnothing}, R_{1}=D_{J}, R_{2}=D_{J^{\prime}}$, and $R_{0}=D_{J \cup J^{\prime}}$. Since $v(f) \geq 0$ for each $f \in K\left[z_{j} \mid j \in J \cup J^{\prime}\right][X, Y]$, we also have $v(f) \geq 0$ for each $f$ in the completion $R_{0}$. The ring $R$ is complete with respect to $v$, and $R=R_{1}+R_{2}$ by Proposition 1.7. Let $\mathfrak{p}, \mathfrak{p}_{1}, \mathfrak{p}_{2}$, and $\mathfrak{p}_{0}$ be the centers of $v$ at $R, R_{1}, R_{2}$, and $R_{0}$, respectively. Then $\mathfrak{p}$ is generated by $X$ and $Y$, and $\mathfrak{p}_{0}$ is generated by $X-c_{j} Y$ for any $j \in J$, by Proposition 1.5. It follows that $\mathfrak{p} R_{0}=\mathfrak{p}_{0}$. In order to apply Lemma 1.8 , it remains to check that $R_{1} / \mathfrak{p}_{1} \cap R_{2} / \mathfrak{p}_{2}=R / \mathfrak{p}$ in $R_{0} / \mathfrak{p}_{0}$. Indeed, we have $R_{1} / \mathfrak{p}_{1}=K\left[z_{j} \mid j \in J\right], R_{2} / \mathfrak{p}_{2}=K\left[z_{j} \mid j \in J^{\prime}\right]$, and $R_{0} / \mathfrak{p}_{0}=K$. By Lemma 1.9, we are done. 
Proposition 1.11. For each $i \in I, Q_{i}^{\prime} \cap Q_{i}=E$.

Proof. Since $Q_{i}^{\prime}=\bigcap_{j \neq i} Q_{j}$, the assertion is that $\bigcap_{j \in I} Q_{j}=E$. Indeed, let $y \in \bigcap_{j \in I} Q_{j}$. For each $j \in J$ write $y=f_{j} / q_{j}$ with $f_{j} \in D_{I \backslash\{j\}}, q_{j} \in K \llbracket X, Y \rrbracket$. Taking a common denominator we may assume that $q_{j}$ is independent of $j$, and denote $q=q_{j}$ (for all $j \in J$ ). It suffices to prove that $q y \in K \llbracket X, Y \rrbracket \subseteq E$. But $q y=q_{j} y=f_{j} \in D_{I \backslash\{j\}}$ for all $j \in I$; hence, by Proposition 1.10,

$$
q y \in \bigcap_{j \in I} D_{I \backslash\{j\}}=D_{\varnothing}=K \llbracket X, Y \rrbracket .
$$

The next remark gives a rigid-geometric and a formal-geometric interpretation of the rings $D_{J}$.

Remark 1.12. Let $J \subseteq I, j \in J$, and $t=X-c_{j} Y$. By Proposition $1.5, D_{J}=$ $K\left[z_{l} \mid l \in J\right] \llbracket t \rrbracket$ is the $t$-adic completion of $K\left[z_{l} \mid l \in J\right][t]$; thus $D_{J}\left[t^{-1}\right]$ is the $t$-adic completion of $K\left[z_{l} \mid l \in J\right]\left[t, t^{-1}\right]$. We have $K\left[z_{l} \mid l \in J\right]\left[t, t^{-1}\right] \subseteq K((t))\left[z_{l} \mid l \in J\right]$ $\subseteq D_{J}\left[t^{-1}\right]$; hence $D_{J}\left[t^{-1}\right]$ is the $t$-adic completion of $A:=K((t))\left[z_{l} \mid l \in J\right]$. Let $T=K \llbracket t \rrbracket, F=K((t))$, and $s=X / Y$. Then $s$ is a free variable over $F$. Let $v_{t}$ be the $t$-adic valuation on $F$, and extend it to $F(s)$ by $v_{t}(s)=0$. Note that $z_{k}=1 /\left(s-c_{k}\right)$ and $v_{t}\left(c_{l}-c_{k}\right)=0$ for all distinct $l, k \in J$. By [Haran and Jarden 1998, Lemma 3.1(c)] (with $w_{k}, K$, and $x$ there replaced by $z_{k}, F$, and $s$ here), each element $0 \neq f \in A$ can be uniquely written as

$$
f=f_{0}+\sum_{k \in J} \sum_{n=1}^{\infty} f_{k n} z_{k}^{n},
$$

where $f_{0}, f_{k n} \in F$ are almost all zero. Uniqueness in the presentation (1) implies that $v_{t}(f)=\min _{k n}\left\{v_{t}\left(f_{0}\right), v_{t}\left(f_{k n}\right)\right\}$.

By [Haran and Jarden 1998, Lemma 3.3] the completion $D_{J}\left[t^{-1}\right]$ of $A$ is the ring of holomorphic functions on the affinoid $U=\mathbb{P} \backslash\left(\bigcup_{l \in J} B\left(c_{l}\right)\right)$, where $\mathbb{P}$ is the projective $s$-line and $B\left(c_{l}\right)$ is a disc of radius 1 with center $c_{l}$ for each $l \in J$ [Fresnel and van der Put 2004, §2.2]. Moreover, each element $f \in D_{J}\left[t^{-1}\right]$ can be uniquely presented as in (1), where $f_{0} \in F$ and $\left\{f_{l n}\right\}_{n=1}^{\infty}$ is a null sequence in $F$ (with respect to $v_{t}$ ) for each $l \in J$. Thus, in the rigid-geometric language, $D_{J}$ is the ring of holomorphic functions on $U$ having no pole at $t$. Its elements are of the form (1), where the coefficients are now in $T$ (and $\left\{f_{k n}\right\}_{n=1}^{\infty}$ is a null sequence for each $k \in J)$. In particular, $T\left[z_{l} \mid l \in J\right]$ is dense in $D_{J}$.

Let $\hat{X}$ be the projective $s$-line over $T$, and let $X$ be its closed fibre. Put

$$
U=X \backslash\left\{c_{l} \mid l \in J\right\} .
$$

Then $R_{U}=T\left[1 /\left(s-c_{l}\right) \mid l \in J\right]=T\left[z_{l} \mid l \in J\right]$ is the set of functions on $\hat{X}$ which are regular on $U$. Since $R_{U}$ is $t$-adically dense in $D_{J}=K\left[1 /\left(s-c_{l}\right) \mid l \in J\right] \llbracket t \rrbracket$, 
$D_{J}$ is the $t$-adic completion of $R_{U}$. In formal-geometric language, this means that $D_{J}=\hat{R}_{U}$ is the ring of regular functions on the $t$-adic thickening of $U$ [Harbater and Hartmann 2007, Notation 4.3].

Corollary 1.13. Let $J \subseteq I, j \in J$.

(a) For each $0 \neq g \in D_{J}$,

$$
D_{J}\left[\left(X-c_{j} Y\right)^{-1}\right]=K\left(\left(X-c_{j} Y\right)\right)\left[z_{k} \mid k \in J\right]+g D_{J}\left[\left(X-c_{j} Y\right)^{-1}\right] .
$$

(b) For each $f \in D_{J}$ there are $h \in K \llbracket X-c_{j} Y \rrbracket\left[z_{j}\right]$ and $u \in D_{J}^{\times}$such that $f=h u$.

(c) The ring $Q_{j}$ is a field.

Proof. In the notation of Remark 1.12, each element $f \in D_{J}\left[t^{-1}\right]$ can be written in the form $u \cdot h$ with $u \in D_{J}\left[t^{-1}\right]^{\times}$and $h \in F\left[z_{j}\right]$, by [Haran and Jarden 1998, Lemma 3.7]. If $f \in D_{J}$ then we can multiply $u$ and $h$ by a power of $t$ to assume that $u \in D_{J}^{\times}$and $h \in K \llbracket t \rrbracket\left[z_{j}\right]$. This proves (b). Part (a) is given by [Haran and Jarden 1998, Corollary 3.8]. By [Harbater and Hartmann 2007, Corollary 4.8] (now viewing the rings $D_{J}$ in the formal-geometric context) Quot $D_{J}$ is the compositum of $K((t))(X / Y)$ and $D_{J}$. Since $K((t))(X / Y) \subseteq E$, we have Quot $D_{J}=E D_{J}$. Applying this argument for $J \backslash\{j\}$ instead of $J$ we have $Q_{j}=\operatorname{Quot}\left(D_{I \backslash\{j\}}\right)=$ $E D_{I \backslash\{j\}}$; hence Quot $Q_{j}=\operatorname{Quot}\left(E D_{I \backslash\{j\}}\right)=E \operatorname{Quot}\left(D_{I \backslash\{j\}}\right)=E D_{I \backslash\{j\}}=Q_{j}$ is a field.

The proof of the following proposition is based on that of Corollary 4.4 of [Haran and Jarden 1998]. (We cannot use that corollary as it is, since condition $\left(\mathrm{e}^{\prime}\right)$ of that claim does not hold for $D_{I}$ itself.)

Proposition 1.14. Let $i \in I, n \in \mathbb{N}$, and $b \in \mathrm{GL}_{n}(Q)$. There exist $b_{1} \in \mathrm{GL}_{n}\left(Q_{i}\right)$ and $b_{2} \in \mathrm{GL}_{n}\left(Q_{i}^{\prime}\right)$ such that $b=b_{1} \cdot b_{2}$.

Proof. Denote by $|\cdot|$ the absolute value on $Q$ that corresponds to $v$. Each of the rings $A=D_{I}, A_{1}=D_{I \backslash\{i\}}$, and $A_{2}=D_{\{i\}}$ is complete with respect to $|\cdot|$ and Proposition 1.7 asserts that condition $\left(\mathrm{d}^{\prime}\right)$ of Example 4.3 of [Haran and Jarden 1998] holds for these rings. We extend $|\cdot|$ to the maximum norm $\|\cdot\|$ on $M_{n}(Q)$, as in the same example. Then $M_{n}(A), M_{n}\left(A_{1}\right)$, and $M_{n}\left(A_{2}\right)$ are complete with respect to $\|\cdot\|$ and condition (d) of [Haran and Jarden 1998, §4] holds. By Cartan's lemma [Haran and Jarden 1998, Lemma 4.2], for each $a \in \mathrm{GL}_{n}(A)$ with $\|a-1\|<1$ there exist $a_{1} \in \mathrm{GL}_{1}\left(A_{1}\right)$ and $a_{2} \in \mathrm{GL}_{1}\left(A_{2}\right)$ such that $a=a_{1} \cdot a_{2}$.

Let $E_{1}=$ Quot $A_{1}=Q_{i}$ and $E_{2}=$ Quot $A_{2}=Q_{i}^{\prime}$. In order to factor $b$ (which need not be in $\mathrm{GL}_{n}(A)$ ), let $t=X-c_{i} Y, T=k \llbracket t \rrbracket$. By Remark 1.12 (for $J=I$ ) $A_{0}=T\left[z_{k} \mid k \in I\right]$ is a dense subring of $A$, and by Corollary 1.13(b) there exists $h \in A_{0}$ such that $h b \in \mathrm{M}_{n}(A)$. If $h b=b_{1} b_{2}^{\prime}$ with $b_{1} \in \mathrm{GL}_{n}\left(E_{1}\right)$ and $b_{2}^{\prime} \in \mathrm{GL}_{n}\left(E_{2}\right)$, then $b=b_{1} b_{2}$ with $b_{2}=b_{2}^{\prime} / h \in \mathrm{GL}_{n}\left(E_{2}\right)$. So we may assume that $b \in \mathrm{M}_{n}(A)$. 
Let $0 \neq d=\operatorname{det}(b) \in A$. By Corollary 1.13(b) there are $0 \neq g \in A_{0}$ and $u \in A^{\times}$ such that $d=g u$. Let $b^{\prime \prime} \in \mathrm{M}_{n}(A)$ be the adjoint matrix of $b$, so that $b b^{\prime \prime}=d 1$. Let $b^{\prime}=u^{-1} b^{\prime \prime}$. Then $b^{\prime} \in \mathrm{M}_{n}(A)$ and $b b^{\prime}=g 1$. Put

$$
V=\left\{a^{\prime} \in \mathrm{M}_{n}\left(A\left[t^{-1}\right]\right) \mid b a^{\prime} \in g M_{n}\left(A\left[t^{-1}\right]\right)\right\}, \quad V_{0}=V \cap M_{n}\left(A_{0}\left[t^{-1}\right]\right) .
$$

$V$ is an additive subgroup of $M_{n}\left(A\left[t^{-1}\right]\right)$ and $g M_{n}\left(A\left[t^{-1}\right]\right) \leq V$. By Corollary 1.13(a), $M_{n}\left(A\left[t^{-1}\right]\right)=M_{n}\left(A_{0}\left[t^{-1}\right]\right)+g M_{n}\left(A\left[t^{-1}\right]\right)$; hence $V=V_{0}+g M_{n}\left(A\left[t^{-1}\right]\right)$.

Since $A_{0}$ is dense in $A, g M_{n}\left(A_{0}\left[t^{-1}\right]\right)$ is dense in $g M_{n}\left(A\left[t^{-1}\right]\right)$. It follows that $V_{0}=V_{0}+g M_{n}\left(A_{0}\left[t^{-1}\right]\right)$ is dense in $V=V_{0}+g M_{n}\left(A\left[t^{-1}\right]\right)$. As $b^{\prime} \in V$, there exists $a_{0} \in V_{0}$ such that $\left\|b^{\prime}-a_{0}\right\|<|g| /\|b\|$. Put $a=a_{0} / g \in M_{n}(Q)$. Then $b a \in M_{n}\left(A\left[t^{-1}\right]\right)$ and $\|1-b a\|=\left\|(1 / g) b\left(b^{\prime}-a_{0}\right)\right\| \leq(1 /|g|)\|b\| \cdot\left\|b^{\prime}-a_{0}\right\|<1$. Hence $\|b a\|=1$, so each entry in $b a$ has a nonnegative value at $v$. By Remark 1.12, $v$ coincides with the $t$-adic valuation on $A$; hence all the entries of $b a$ belong to $A$. Thus $b a \in M_{n}(A)$, and since $\|1-b a\|<1$ and $M_{n}(A)$ is complete, $b a \in \mathrm{GL}_{n}(A)$. In particular, det $a \neq 0$ and hence $a \in \mathrm{GL}_{n}\left(\right.$ Quot $\left.A_{0}\right) \subseteq \mathrm{GL}_{n}\left(E_{2}\right)$. By the first paragraph of this proof, there exist $b_{1} \in \mathrm{GL}_{n}\left(A_{1}\right) \subseteq \mathrm{GL}_{n}\left(E_{1}\right)$ and $b_{2}^{\prime} \in \mathrm{GL}_{n}\left(A_{2}\right)$ such that $b a=b_{1} b_{2}^{\prime}$. Then $b_{2}=b_{2}^{\prime} a^{-1} \in \mathrm{GL}_{n}\left(E_{2}\right)$ satisfies $b=b_{1} b_{2}$.

Corollary 1.15. Suppose $G$ is a finite group. For each $i \in I$ let $F_{i}$ be a Galois extension of $E$ with group $G_{i}$ contained in $G$, such that $F_{i} \subseteq Q_{i}^{\prime}$. If $G=\left\langle G_{i} \mid i \in I\right\rangle$ then $\mathscr{E}=\left(E, F_{i}, Q_{i}, Q ; G_{i}, G\right)_{i \in I}$ is a patching datum [Haran and Jarden 1998, Definition 1.1]. In particular, $G$ occurs as a Galois group over $E$.

Proof. By Corollary 1.13(c), $Q_{i}$ is a field for each $i \in I$. Conditions (2a), (2b), and (2d) of [Haran and Jarden 1998, Definition 1.1] are given in the hypothesis. Conditions (2c) and (2e) are given by Propositions 1.11 and 1.14, respectively. Thus $\mathscr{E}$ is a patching datum. By [Haran and Jarden 1998, Lemma 1.3(a)], there exists a Galois extension $F$ of $E$ with group $G$.

\section{2. $p$-groups}

Fix the notation of Section 1, including that of Construction 1.1, and let $p$ denote a prime number. In this section we realize $p$-groups of rank at most 2 by adequate extensions of $E$, and embed these extensions into the analytic fields.

Lemma 2.1. Let $J \subseteq I, j \in J$, and $t=X-c_{j} Y$.

(a) Suppose $f=\sum_{l=0}^{d} f_{l} z_{j}^{l} \in K \llbracket t \rrbracket\left[z_{j}\right]$ is a polynomial such that $v\left(f_{1}\right)=0$ and $v\left(f_{l}\right)>0$ for each $l>1$. Then $f$ is prime in $D_{J}\left[t^{-1}\right]$.

(b) The ring $D_{J}\left[t^{-1}\right]$ is a unique factorization domain.

(c) For each $a, b, c \in K^{\times}$with $a \neq-b$ and $2 \leq m \in \mathbb{N}$, the elements $1+a z_{j}+$ $t^{m-1} z_{j}^{m}, 1+b z_{j}-t^{m-1} z_{j}^{m}, 1+c z_{j}$ are nonassociate primes of $D_{J}\left[t^{-1}\right]$. 
Proof. Set $F=K((t))$. Then $D_{J}\left[t^{-1}\right]=F\left\{z_{k} \mid k \in J\right\}$ (see Remark 1.12). Viewed as an element of $F\left\{z_{j}\right\}, f$ is regular of pseudodegree 1; see Definition 1.4 of [Haran and Völklein 1996]. Hence, by Corollary 1.7 of the same work, we have $f=u \cdot q$, where $u \in F\left\{z_{j}\right\}^{\times} \subseteq D_{J}\left[t^{-1}\right]^{\times}$and $q=q_{0}+z_{j} \in F\left[z_{j}\right]$ is a linear polynomial with $v\left(q_{0}\right) \geq 0$. Thus to prove (a), it suffices to show that $q$ is prime in $D_{J}\left[t^{-1}\right]$. Without loss of generality $q_{0} \neq 0$, and we set $c=c_{j}-1 / q_{0}$. Then $q=z_{j}-1 /\left(c-c_{j}\right)$; hence, by [Paran 2008, Lemma 6.4(a)] (with $D, r$, and 1 there replaced by $F, 1$, and $j$ here), $q$ generates the kernel of an epimorphism from $D_{J}\left[t^{-1}\right]$ onto a domain (actually a field here); hence $q$ is prime. This proves (a).

Since $D_{J}\left[t^{-1}\right]$ is a principal ideal domain by [Haran and Jarden 1998, Proposition 3.9], part (b) follows.

By part (a), $r=1+a z_{j}+t^{m-1} z_{j}^{m}, r^{\prime}=1+b z_{j}-t^{m-1} z_{j}^{m}$, and $s=1+c z_{j}$ are primes of $D_{J}\left[t^{-1}\right]$. If $s \mid r$, then $-1 / c$ is a root of $r$, a contradiction. Thus $r$ and $s$ (and similarly, $r^{\prime}$ and $s$ ) are nonassociates.

If $r \mid r^{\prime}$ then $r \mid r+r^{\prime}$. By the argument of the preceding paragraph, $r+r^{\prime}=$ $2+(a+b) z_{j}$ is a prime, nonassociate to $r$, a contradiction. This proves (c).

Lemma 2.2. Let $K$ be a field that contains a primitive $q$-th root of unity, for some $q \in \mathbb{N}$. Let $v$ be a discrete valuation on $K$ which is trivial on the prime field of $K$, and let $a \in K$ with $v(a)=0$. Suppose $L=K\left(a^{1 / q}\right)$ is a Kummer extension of $K$, and that $L / K$ is unramified at $v$. Then $v\left(x^{\sigma}\right)=v(x)$ for each $x \in L$ and $\sigma \in \operatorname{Gal}(L / K)$.

Proof. Extend $v$ arbitrarily to $L$, let $O$ be the valuation ring of $v$ in $K$, and $O^{\prime}$ the valuation ring of $v$ in $L$. Since $K$ contains a primitive $q$-th root of unity, $q$ is not divisible by $p=\operatorname{char} K$. Thus $d=\operatorname{disc}\left(T^{q}-a, K\right)=k a^{q-1}$, where $k \in \mathbb{Z}$ is not divisible by $p$. Hence $v(d)=0$, and by [Fried and Jarden 2005, Lemma 6.1.2] we have $O^{\prime}=O\left[a^{1 / q}\right]$. Put $\alpha=a^{1 / q}$ and let $x=\sum_{i=0}^{q-1} b_{i} \alpha^{n} \in K$, with $b_{0}, \ldots, b_{q-1} \in K$. We claim that $v(x)=\min _{i} v\left(b_{i}\right)$. Indeed, since $L / K$ is unramified at $v$, we may multiply $x$ by a power of a uniformizer of $v$ in $K$, to assume that $v(x)=0$. Since $O^{\prime}=O[\alpha], v\left(b_{i}\right) \geq 0$ for each $0 \leq i \leq q-1$. On the other hand $v(x) \geq \min _{i} v\left(b_{i} \alpha^{i}\right)=\min _{i} v\left(b_{i}\right)$, since $v(\alpha)=(1 / n) v(a)=0$. Thus $v\left(b_{i}\right)=0$ for some $0 \leq i \leq q-1$; hence $v(x)=\min _{i} v\left(b_{i}\right)$.

Now, let $\sigma \in \operatorname{Gal}(L / K)$ and let $x=\sum_{i=0}^{q-1} b_{i} \alpha^{n} \in K$, with $b_{0}, \ldots, b_{q-1} \in K$, be an arbitrary element. We have $\alpha^{\sigma}=\zeta \alpha$, where $\zeta$ is some $q$-th root of unity. Then $v\left(x^{\sigma}\right)=v\left(\sum_{i=0}^{q-1} b_{i} \zeta^{i} \alpha^{i}\right)=\min _{i} v\left(b_{i} \zeta^{i}\right)=\min _{i} v\left(b_{i}\right)=v(x)$.

Recall that given a field $K$, any $K$-central simple algebra $A$ is of the form $\mathrm{M}_{n}(D)$ for some $K$-division algebra $D$. The index of $A$ is defined to be ind $A=\sqrt{\operatorname{dim}_{K} D}$. So, $A$ is a division algebra if and only if ind $A=\sqrt{\operatorname{dim}_{K} A}$. Let us denote Brauer equivalence by $\sim$ and the exponent of $A$ (its order in the Brauer group) by $\exp A$. A subfield $F$ of $A$ is a maximal subfield of $A$ if and only if $\operatorname{dim}_{K} A=[F: K]^{2}$. 
Furthermore, a field $F$ is a maximal subfield of $A$ if and only if $\operatorname{dim}_{K} A=[F: K]^{2}$ and $F$ splits $A$ [Reiner 1975, Theorem 28.4 and Corollary 28.11].

The proof of the next proposition is partially based on that of [Harbater et al. 2009, Proposition 4.4].

Proposition 2.3. Fix $i \in I$, and let $H$ be an abelian p-group of rank at most 2, where $p \neq$ char $K$. Suppose $K$ contains an $|H|$-th primitive root of unity. Let $E^{\prime}$ be a finite extension of $E$. Then there exists an $H$-Galois extension $F_{i} / E$ such that $F_{i} \subseteq Q_{i}^{\prime}, F_{i}$ is contained as a maximal subfield in an E-division algebra $D_{i}^{\prime}$, and $D_{i}^{\prime} \otimes_{E} E^{\prime} Q_{i}$ remains a division algebra (where $E^{\prime} Q_{i}$ is the compositum of $E^{\prime}$ and $Q_{i}$ in an algebraic closure of $Q$ ).

Proof. Let us start by constructing $F_{i}$. Write $H=C_{q} \times C_{q}^{\prime}$, where $q$ and $q^{\prime}$ are powers of $p$. For each $k \in \mathbb{N}$, the elements $X-c_{i} Y+Y^{k}$ and $X+c_{i} Y-Y^{k}$ are irreducible and hence prime in the unique factorization domain $K \llbracket X, Y \rrbracket$. Only finitely many primes of $K \llbracket X, Y \rrbracket$ are ramified at $E^{\prime} / E$; hence for a sufficiently large $2 \leq k \in \mathbb{N}$, $f=X-c_{i} Y+Y^{k}$, and $g=X+c_{i} Y-Y^{k}$ are unramified at $E^{\prime} / E$. That is, the corresponding valuations $v_{f}$ and $v_{g}$ are unramified. Let $a=f /\left(X-c_{i} Y\right)$ and $b=g /\left(X-c_{i} Y\right)$. Clearly $v_{f}\left(X-c_{i} Y\right)=v_{f}(g)=0$; hence $v_{f}(a)=1$ and $v_{f}(b)=0$. Similarly, $v_{g}(a)=0$ and $v_{g}(b)=1$. Consider the polynomial $h(U)=U^{q}-a$ over $D_{\{i\}}=K\left[z_{i}\right] \llbracket X-c_{i} Y \rrbracket$. Note that $a=1+z_{i}^{k}\left(X-c_{i} Y\right)^{k-1}$, hence $h(1) \in\left(X-c_{i} Y\right) D_{\{i\}}$ and $h^{\prime}(1)=q \in K^{\times} \subseteq D_{\{i\}}^{\times}$. By the ring version of Hensel's lemma (for the ideal $\left.\left(X-c_{i} Y\right) D_{\{i\}}\right) h(U)$ has a root $s \in D_{\{i\}}$. Note that $v_{f}(s)=1 / q \notin \mathbb{Z}$; hence $s \notin E$. Since $K$ contains a primitive $|H|$-th root of unity, it contains a primitive $q$-th root of unity. By Kummer theory $E(s) / E$ is a Galois extension with group $C_{q}$. Similarly, there exists $s^{\prime} \in D_{\{i\}}$ satisfying $\left(s^{\prime}\right)^{q^{\prime}}=b$, and $E\left(s^{\prime}\right) / E$ is Galois with group $C_{q^{\prime}}$. Let $F_{i}=E\left(s, s^{\prime}\right) \subseteq Q_{i}^{\prime}$.

Since $v_{f}(a)=1, h(U)$ is irreducible over $E$, by Eisenstein's criterion. Denoting the reduction modulo $g$ by $\bar{h}, \bar{h}(U)=U^{q}-\bar{a}$ is separable, since $\bar{a} \neq 0$. Thus by [Fried and Jarden 2005, Lemma 2.3.4], $E(s) / E$ is unramified at $v_{g}$. Clearly, $E\left(s^{\prime}\right) / E$ is totally ramified at $v_{g}$. Thus $E(s)$ and $E\left(s^{\prime}\right)$ are linearly disjoint over $E$; hence $\operatorname{Gal}\left(F_{i} / E\right)=H$.

Let $D_{i}^{\prime}$ be the quaternion algebra $(a, b)_{q q^{\prime}}$ [Pierce 1982, Section 15.4]. Note that $D_{i}^{\prime}$ can be also viewed as the cyclic algebra $\left(E\left(a^{1 / q q^{\prime}}\right) / E, \sigma, b\right)$, for some generator $\sigma$ of $\operatorname{Gal}\left(E\left(a^{1 / q q^{\prime}}\right) / E\right)$. We claim that $F_{i}$ splits $D_{i}^{\prime}$. By [Reiner 1975, Theorem 30.8], we have

$$
D_{i}^{\prime} \otimes_{E} E(s) \sim\left(E\left(s^{1 / q^{\prime}}\right) / E(s), \sigma^{q}, b\right) ;
$$

thus $D_{i}^{\prime} \otimes_{E} F_{i} \sim\left(F_{i}\left(s^{1 / q^{\prime}}\right) / F_{i}, \sigma^{q}, b\right)$. The cyclic algebra $\left(F_{i}\left(s^{1 / q^{\prime}}\right) / F_{i}, \sigma^{q}, b\right)$ is split if and only if $b$ is a norm from $F_{i}\left(s^{1 / q^{\prime}}\right)$ (see for example [Reiner 1975, Theorem 30.4]), i.e., if and only if $b \in N_{F_{i}\left(s^{1 / q^{\prime}}\right) / F_{i}}\left(F_{i}\left(s^{1 / q^{\prime}}\right)\right)$. This holds since 
$b=N_{F_{i}\left(s^{1 / q^{\prime}}\right) / F_{i}}\left(s^{\prime}\right)$. Thus $F_{i}$ splits $D_{i}^{\prime}$. As $\left[F_{i}: E\right]=q q^{\prime}, F_{i}$ is a maximal subfield of the $E$-central simple algebra $D_{i}^{\prime}$. We shall show that $D_{i}^{\prime} \otimes_{E} E^{\prime} Q_{i}$ is a division algebra and from this it will follow that $D_{i}^{\prime}$ is a division algebra.

In order to show that $D_{i}^{\prime} \otimes_{E} E^{\prime} Q_{i}$ is a division algebra we construct auxiliary valuations. Choose $j \in I \backslash\{i\}$, and let

$$
t=X-c_{j} Y, \quad r=1+\left(c_{j}+c_{i}\right) z_{j}-t^{k-1} z_{j}^{k}, \quad r^{\prime}=1+\left(c_{j}-c_{i}\right) z_{j}+t^{k-1} z_{j}^{k} .
$$

By Lemma 2.1(c) $r$ and $r^{\prime}$ are nonassociate prime elements in $D_{I \backslash\{i\}}\left[t^{-1}\right]$, so they define discrete valuations $v_{r}$ and $v_{r^{\prime}}$ on $Q_{i}=\operatorname{Quot}\left(D_{I \backslash\{i\}}\right)=\operatorname{Quot}\left(D_{I \backslash\{i\}}\left[t^{-1}\right]\right)$ such that $v_{r}\left(r^{\prime}\right)=v_{r^{\prime}}(r)=0$. By Lemma 2.1(c) we also have

$$
v_{r^{\prime}}\left(1+\left(c_{j}-c_{i}\right) z_{j}\right)=v_{r}\left(1+\left(c_{j}-c_{i}\right) z_{j}\right)=0 .
$$

Note that

$$
b=\frac{X-c_{j} Y+\left(c_{j}+c_{i}\right) Y-Y^{k}}{X-c_{j} Y+\left(c_{j}-c_{i}\right) Y}=\frac{t+\left(c_{j}+c_{i}\right) t z_{j}-t^{j} z_{j}^{k}}{t+\left(c_{j}-c_{i}\right) t z_{j}}=\frac{r}{1+\left(c_{j}-c_{i}\right) z_{j}} .
$$

Similarly, $a=r^{\prime} /\left(1+\left(c_{j}-c_{i}\right) z_{j}\right)$. Thus $v_{r}(b)=1, v_{r^{\prime}}(b)=0, v_{r}(a)=0$, and $v_{r^{\prime}}(a)=1$. Then the polynomial $U^{q q^{\prime}}-a$ is irreducible over $D_{I \backslash\{i\}}$, by Eisenstein's criterion (using $v_{r^{\prime}}$ ). Thus $Q_{i}\left(a^{1 / q q^{\prime}}\right) / Q_{i}$ is unramified at $v_{r}$ (again by [Fried and Jarden 2005, Lemma 2.3.4]); hence so is $E^{\prime} Q_{i}\left(a^{1 / q q^{\prime}}\right) / E^{\prime} Q_{i}$.

Only finitely many primes of the unique factorization domain $D_{I \backslash\{i\}}\left[t^{-1}\right]$ (see Lemma 2.1(b)) are ramified at the finite extension $E^{\prime} Q_{i} / Q_{i}$; hence, without loss of generality, we may assume that $E^{\prime} Q_{i} / Q_{i}$ is unramified at $v_{r^{\prime}}$ (by possibly choosing an even larger $k$ beforehand). On the other hand, $Q_{i}\left(a^{1 / q q^{\prime}}\right) / Q_{i}$ is totally ramified at $v_{r^{\prime}}$; hence

$$
\left[E^{\prime} Q_{i}\left(a^{1 / q q^{\prime}}\right): E^{\prime} Q_{i}\right]=\left[Q_{i}\left(a^{1 / q q^{\prime}}\right): Q_{i}\right]=q q^{\prime} .
$$

We can now show that $D_{i}^{\prime} \otimes_{E} E^{\prime} Q_{i}$ is a division algebra. A sufficient condition for this to hold is that $\exp \left(D_{i}^{\prime} \otimes_{E} E^{\prime} Q_{i}\right)=q q^{\prime}$. This happens if and only if for every $1 \leq m \leq q q^{\prime}-1$ the algebra $\left(E^{\prime} Q_{i}\left(a^{1 / q q^{\prime}}\right) / E^{\prime} Q_{i}, \sigma, b^{m}\right) \sim\left(D_{i}^{\prime} \otimes_{E} E^{\prime} Q_{i}\right)^{m}$ does not split. Let $N$ denote the norm $N_{E^{\prime}} Q_{i}\left(a^{1 / q q^{\prime}}\right) / E^{\prime} Q_{i}$. For any $1 \leq m \leq q q^{\prime}-1$, the algebra $\left(D_{i}^{\prime} \otimes_{E} E^{\prime} Q_{i}\right)^{m}$ splits if and only if $b^{m} \in N\left(E^{\prime} Q_{i}\left(a^{1 / q q^{\prime}}\right)^{\times}\right)$[Reiner 1975, Theorem 30.4].

Since $E^{\prime} Q_{i}\left(a^{1 / q q^{\prime}}\right) / E^{\prime} Q_{i}$ is unramified at $v_{r}$, we have $v_{r}(x)=v_{r}\left(x^{\sigma}\right)$ for each $x \in E^{\prime} Q_{i}\left(a^{1 / q q^{\prime}}\right)$, by Lemma 2.2. Hence

$$
v_{r}(N(x))=\sum_{l=0}^{q q^{\prime}-1} v_{r}\left(x^{\sigma^{l}}\right)=q q^{\prime} v_{r}(x)
$$

for all $x \in E^{\prime} Q_{i}\left(a^{1 / q q^{\prime}}\right)$. Since $v_{r}(b)=1, b^{m} \notin N\left(E^{\prime} Q_{i}\left(a^{1 / q q^{\prime}}\right)^{\times}\right)$for all $1 \leq m \leq$ $q q^{\prime}-1$. Thus, $\exp \left(D_{i}^{\prime} \otimes_{E} E^{\prime} Q_{i}\right)=q q^{\prime}$ and $D_{i}^{\prime} \otimes_{E} E^{\prime} Q_{i}$ is a division algebra. 


\section{Patching and admissibility}

We have established the patching machinery needed to prove our Main Theorem. We first recall some general properties of induced algebras and Frobenius algebras.

Remark 3.1 (induced algebras). Let $G$ be a finite group and $H \leq G$. Let $P / Q$ be a finite field extension with $H=\operatorname{Gal}(P / Q)$. Let

$$
N=\operatorname{Ind}_{H}^{G} P=\left\{\sum_{\theta \in G} a_{\theta} \theta \mid a_{\theta} \in P, a_{\theta}^{\tau}=a_{\theta \tau} \text { for all } \theta \in G, \tau \in H\right\}
$$

be a ring with respect to point-wise addition and multiplication. Then $P$ can be embedded as a subring of $N$ by choosing representatives $\theta_{1}=1, \ldots, \theta_{k}$ of $H \backslash G$ and sending an element $x \in P$ to $\sum_{i=1, \tau \in H}^{k} x^{\tau} \theta_{i} \tau$. Furthermore, by choosing different representatives $N$ can be presented as a direct sum of copies of $P$.

If $P$ splits a central simple $Q$-algebra $A$ then

$$
\operatorname{Ind}_{H}^{G} P \otimes_{Q} A \cong \operatorname{Ind}_{H}^{G} P \otimes_{P}\left(P \otimes_{Q} A\right) \sim \operatorname{Ind}_{H}^{G} P \otimes_{P} P \cong \operatorname{Ind}_{H}^{G} P ;
$$

hence $A$ splits over $\operatorname{Ind}_{H}^{G} P$. For a definition of a split separable (Azumaya) algebra over a ring, see [DeMeyer and Ingraham 1971, §5].

The next definition, remark, and proposition appear in Section 2.1 of [Jacobson 1996].

Definition 3.2 (Frobenius algebras). Let $F$ be a field. An $F$-algebra $A$ is a Frobenius algebra if $A$ contains a hyperplane that does not contain any nonzero one sided ideal of $A$.

Remark 3.3. An algebra $A_{1} \oplus \cdots \oplus A_{s}$ is Frobenius if and only if $A_{i}$ is Frobenius for each $1 \leq i \leq s$. Any algebra $F[a]$ (with a single generator) is Frobenius. Let $L / K$ be an $H$-extension. By Remark 3.1, $\operatorname{Ind}_{H}^{G} L$ can be decomposed into a sum of copies of $L$ and it follows that $\operatorname{Ind}_{H}^{G} L$ is a Frobenius algebra.

Proposition 3.4 [Jacobson 1996, Theorem 2.2.3]. Let A be an F-central simple algebra and $K$ a commutative Frobenius subalgebra of $A$ such that $\operatorname{dim}_{F} A=$ $[K: F]^{2}$. Then any embedding of $K$ into $A$ can be extended to an inner automorphism of $A$.

Lemma 3.5. Let $R$ be an equicharacteristic complete local domain of dimension $r$. Suppose that the residue field of $R$ contains a primitive root of unity of order $k$, for each $k \in \mathbb{N}$ with char $R \backslash k$. Then $R$ is a finite module over a subring of the form $K \llbracket X_{1}, \ldots, X_{r} \rrbracket$, where $K$ is a field containing a primitive root of unity of order $k$, for each $k \in \mathbb{N}$ with char $K \backslash k$.

Proof. By Cohen's structure theorem [Matsumura 1986, §29], $R$ is finitely generated over a subring of the form $B=K_{0} \llbracket X_{1}, \ldots, X_{n} \rrbracket$, for some field $K_{0}$. Since $\operatorname{dim} B=\operatorname{dim} R=r$, we have $n=r$. 
Let $K$ be the field obtained by adjoining all primitive roots of unity of order not divisible by char $R$ to $K_{0}$. By our assumptions, $K$ is contained in the residue field of $R$; hence $K / K_{0}$ is a finite (separable) extension. By Hensel's lemma, $R$ contains $K$. Since $\left[K: K_{0}\right]$ is finite, $K\left(K_{0} \llbracket X_{1}, \ldots, X_{r} \rrbracket\right)=K \llbracket X_{1}, \ldots, X_{r} \rrbracket$. Thus $R$ is finite over $K \llbracket X_{1}, \ldots, X_{r} \rrbracket$ (since it is finite over $K_{0} \llbracket X_{1}, \ldots, X_{r} \rrbracket$ ).

The final ingredient we need in order to prove our Main Theorem is patching of central simple algebras. The content of the next proposition is essentially given in [Harbater et al. 2009], but for specific fields $Q_{i}$, while here we present it for general fields satisfying a matrix factorization property. We note that [Harbater et al. 2009, Theorem 4.1] uses the terminology of categories and equivalence of categories. Here we prefer a more explicit presentation, working with vector spaces and bases, as in [Haran and Jarden 1998]. The proof of the proposition combines the proof of [Haran and Jarden 1998, Lemma 1.2] (where a more restricted assertion is made for specific types of algebras), and the proof of [Harbater and Hartmann 2007, Theorem 7.1(vi)] (where the assertion is made for specific types of fields).

Proposition 3.6. Let $I$ be a finite set. For each $i \in I$ let $Q_{i}$ be a field contained in a $Q_{i}$-algebra $A_{i}$. Let $Q$ be a field containing $Q_{i}$ for each $i \in I$, and contained in a $Q$-algebra $A_{Q}$ which contains $A_{i}$ for each $i \in I$. Moreover, suppose that $A_{i} Q=A_{Q}$ and $\operatorname{dim}_{Q_{i}} A_{i}=\operatorname{dim}_{Q} A_{Q}$ for each $i \in I$. Finally, suppose that

(*) for each $B \in \mathrm{GL}_{n}(Q)$ there exist $B_{i} \in \mathrm{GL}_{n}\left(Q_{i}\right)$ and $B_{i}^{\prime} \in \mathrm{GL}_{n}\left(\bigcap_{j \neq i} Q_{j}\right)$ such that $B=B_{i} B_{i}^{\prime}$.

Then, letting $E=\bigcap_{i \in I} Q_{i}, A=\bigcap_{i \in I} A_{i}$ is an E-algebra satisfying $A Q_{i}=A_{i}$ for each $i \in I$. Moreover, if each $A_{i}$ is central simple, then so is $A$.

Proof. For each $i \in I$, let $\mathscr{C}_{i}$ be a basis for $A_{i}$ over $Q_{i}$. Since $A_{i} Q=A_{Q}$, $\operatorname{Span}_{Q}\left(\mathscr{C}_{i}\right)=A_{Q}$, and since $\operatorname{dim}_{Q_{i}} A_{i}=\operatorname{dim}_{Q} A_{Q}, \mathscr{C}_{i}$ is a basis for $A_{Q}$ over $Q$, for each $i \in I$. We now construct a basis $\mathscr{C}$ for $A_{Q}$ over $Q$, which is also a basis for $A_{i}$ over $Q_{i}$, for all $i \in I$.

For each subset $J$ of $I$ we find, by induction on $|J|$, a basis $\mathscr{V}_{J}$ for $A_{Q}$ over $Q$ which is also a basis for $A_{j}$ over $Q_{j}$, for each $j \in J$. Then for $I=J$ we will get the basis $\mathscr{b}$.

If $J=\varnothing$ there is nothing to prove. Suppose that $|J| \geq 1$, choose $k \in J$ and let $J^{\prime}=J \backslash\{k\}$. By assumption there is a basis $\mathscr{V}_{J}$ for $A_{Q}$ over $Q$ which is a basis for $A_{i}$ over $Q_{i}$ for each $i \in J^{\prime}$. Since $\mathscr{C}_{i}$ is a common basis for $A_{Q}$ and $A_{i}$, there is a matrix $B \in \mathrm{GL}_{n}(Q)$ such that $\mathscr{C}_{k} B=\mathscr{V}_{J^{\prime}}$. By condition $(*)$ in the statement of the proposition, there exist $B_{k} \in \mathrm{GL}_{n}\left(Q_{k}\right)$ and $M \in \mathrm{GL}_{n}\left(\bigcap_{k \neq j \in I} Q_{j}\right) \subseteq$ $\bigcap_{j \in J^{\prime}} \mathrm{GL}_{n}\left(Q_{j}\right)$ such that $B=B_{k} M$. Put $\mathscr{V}_{J}=\mathscr{V}_{J^{\prime}} M^{-1}$. Then $\mathscr{V}_{J}$ is a basis for $A_{Q}$ over $Q$ which is also a basis for $A_{j}$ over $Q_{j}$ for each $j \in J^{\prime}$. Moreover, $\mathscr{V}_{J}$ is also a basis for $A_{k}$ over $Q_{k}$, since $\mathscr{V}_{J}=\mathscr{V}_{k} B M^{-1}=\mathscr{V}_{k} B_{k}$. This completes the induction. 
The existence of the common basis $C$ implies that $A Q_{i}=A_{i}$ for each $i \in I$. As $A_{i}$ is a $Q_{i}$-central simple algebra for any $i \in I$ (a single $i$ suffices), $A$ is an $E$ central simple algebra. This follows, for example, from [Saltman 1999, Theorem 2.2.c].

Proposition 3.7. Let $R$ be an equicharacteristic complete local domain of dimension 2, with residue field containing a primitive root of unity of order $k$ for each $k \in \mathbb{N}$ with char $R \backslash k$. Let $G$ be a finite group of order not divisible by char $R$, whose Sylow subgroups are abelian of rank at most 2. Then $G$ is admissible over Quot $R$.

Proof. By Lemma 3.5, $R$ is a finite module over a subring of the form $B=$ $K \llbracket X, Y \rrbracket$, where $K$ contains a primitive root of unity of order $k$ for each $k \in \mathbb{N}$ not divisible by $p=$ char $R$. Let $E=$ Quot $B=K((X, Y))$ and $E^{\prime}=$ Quot $R$. Then $E^{\prime}$ is a finite extension of $E$.

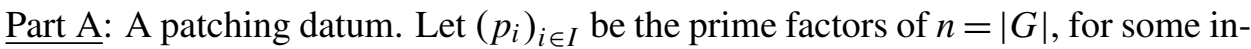
dex set $I$. For each $i \in I$, let $G_{i}$ be a $p_{i}$-Sylow subgroup of $G$. Apply Construction 1.1 to obtain rings $Q_{i}, i \in I$, contained in the common field $Q$. For each $i \in I$, we may apply Proposition 2.3 to obtain a Galois extension $F_{i} / E$ with group $G_{i}$, such that $F_{i} \subseteq Q_{i}^{\prime}$ and $F_{i}$ is contained as a maximal subfield in a division $E$-algebra $D_{i}^{\prime}$. Moreover, $D_{i}^{\prime} \otimes_{E} E^{\prime} Q_{i}$ remains a division algebra. Thus $D_{i}:=D_{i}^{\prime} \otimes_{E} Q_{i}$ is also a division algebra. By Corollary 1.13(c), $Q_{i}$ is a field for each $i \in I$. Put $P_{i}=F_{i} Q_{i}$. Since $F_{i}$ splits $D_{i}^{\prime}, P_{i}$ splits $D_{i}$, and since $\left[P_{i}: Q_{i}\right]=\left[F_{i}: E\right]=\operatorname{ind}\left(D_{i}^{\prime}\right)=\operatorname{ind}\left(D_{i}\right)$, $P_{i}$ is a maximal subfield of $D_{i}$. Let $\mathscr{E}=\left(E, F_{i}, Q_{i}, Q ; G_{i}, G\right)_{i \in I}$. By Corollary $1.15, \mathscr{E}$ is a patching datum.

Part B: Induced algebras [Haran and Jarden 1998, §1]. Consider the induced algebra $N=\operatorname{Ind}_{1}^{G} Q$ of dimension $n$ over $Q$, and the $Q_{i}$-subalgebra $N_{i}=\operatorname{Ind}_{G_{i}}^{G} P_{i}$ for each $i \in I$ (see Remark 3.1). Then $G$ acts on $N$ by $\left(\sum_{\theta \in G} a_{\theta} \theta\right)^{\sigma}=\sum_{\theta \in G} a_{\theta} \sigma^{-1} \theta=$ $\sum_{\theta \in G} a_{\sigma \theta} \theta$ for each $\sigma \in G$. The field $Q$ is embedded diagonally in $N$, which induces an embedding of $Q_{i}$ in $N_{i}$, for each $i \in I$. We view these embeddings as containments. By Lemma 1.2 of [Haran and Jarden 1998] there is a basis for $N$ over $Q$, which is also a basis for $N_{i}$ over $Q_{i}$, for each $i \in I$. In particular, we have $N_{i} Q=N$ for each $i \in I$. By Lemma 1.3 of the same paper, $F=\bigcap_{i \in I} N_{i}$ is a Galois field extension of $E$ with group $G$, and there exists an $E$-embedding of $F$ into $Q$. Denote the image of $F$ under this embedding by $F^{\prime}$.

Part C: Division algebras. It remains to prove that the extension $F^{\prime} / E$ is adequate. Let $A_{Q}=\mathrm{M}_{n}(Q)$, and for each $i \in I$ let $n_{i}=\left[G: G_{i}\right]$. As $A_{Q}$ is split of dimension $n^{2}$ and $N$ is of dimension $n$ over $Q$, we also have an embedding of $N$ into $A_{Q}$. We view $N$ as a subalgebra of $A_{Q}$ via this embedding.

Fix $i \in I$. Since $P_{i}=F_{i} Q_{i}$ splits $D_{i}$, it follows by Remark 3.1 that $N_{i}$ also splits $D_{i}$. Moreover, by [DeMeyer and Ingraham 1971, Theorem 5.5] there is a central 
simple $Q_{i}$-algebra $A_{i}$ which is Brauer equivalent to $D_{i}$, in which $N_{i}$ embeds as a maximal commutative separable $Q_{i}$-subalgebra so that $\operatorname{dim}_{Q_{i}}\left(A_{i}\right)=\operatorname{dim}_{Q_{i}}\left(N_{i}\right)^{2}=$ $n^{2}$. We view $N_{i}$ as contained in $A_{i}$ via this embedding.

Since $P_{i}$ splits $D_{i}$, we have

$$
D_{i} \otimes_{Q_{i}} Q \cong\left(D_{i} \otimes_{Q_{i}} P_{i}\right) \otimes_{P_{i}} Q \cong \mathrm{M}_{n / n_{i}}\left(P_{i}\right) \otimes_{P_{i}} Q \cong \mathrm{M}_{n / n_{i}}(Q) .
$$

Since $\operatorname{ind}\left(D_{i}\right)=n / n_{i}$ and $\operatorname{dim}_{Q} A_{Q}=n^{2}$ we get that $A_{i} \cong M_{n_{i}}\left(D_{i}\right)$. Thus we have $A_{i} \otimes_{Q_{i}} Q \cong M_{n_{i}}\left(D_{i}\right) \otimes_{Q_{i}} Q \cong M_{n}(Q)=A_{Q}$, and we denote the induced $Q$-algebras isomorphism $A_{i} \otimes_{Q_{i}} Q \rightarrow A_{Q}$ by $\psi_{i}$. We cannot identify these two algebras via this isomorphism, since it might not be compatible with the containments $N_{i} \subseteq A_{i}$ and $N \subseteq A_{Q}$. This compatibility problem can be settled similarly to [Harbater et al. 2009, Lemma 4.2]:

By Part B we have $N=N_{i} Q$ and $\operatorname{dim}_{Q_{i}} N_{i}=\operatorname{dim}_{Q} N=n$. Thus we have an isomorphism $\delta_{i}: N=N_{i} Q \rightarrow N_{i} \otimes_{Q_{i}} Q$ for which the following diagram commutes:

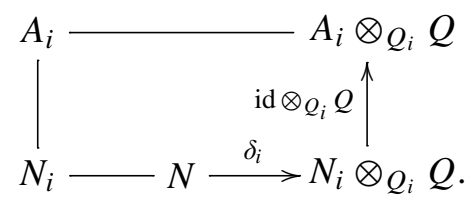

By Remark 3.3, $N=\operatorname{Ind}_{1}^{G} Q$ is a Frobenius (commutative) subalgebra of $A_{Q}$. By Proposition 3.4, the embedding $\psi_{i}\left(\mathrm{id} \otimes_{Q_{i}} Q\right) \delta_{i}: N \rightarrow A_{Q}$ extends to an inner automorphism $\alpha_{i}$ of $A_{Q}$. Let $\psi_{i}^{\prime}=\alpha_{i}^{-1} \psi_{i}$. Then $\alpha_{i}^{-1} \psi_{i}\left(\mathrm{id} \otimes_{Q_{i}} Q\right) \delta_{i}$ is the identity map on $N=N_{i} Q$, so we have the commutative diagram

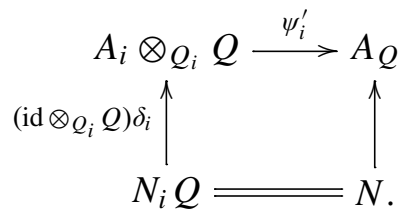

Combining (2) and (3), we get the following commutative diagram:

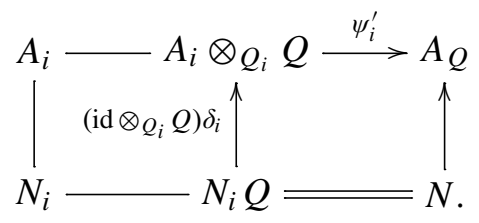

This diagram gives an embedding $A_{i} \rightarrow A_{Q}$ which is compatible with the containments $N_{i} \subseteq A_{i}$ and $N \subseteq A_{Q}$, so we may now identify $A_{i}$ as a subring of $A_{Q}$, via this embedding. Moreover, since $\psi_{i}^{\prime}$ is an isomorphism, we have $A_{i} \otimes_{Q_{i}} Q=A_{i} Q=A_{Q}$ 
by this identification. The following diagram explains the containment relations:

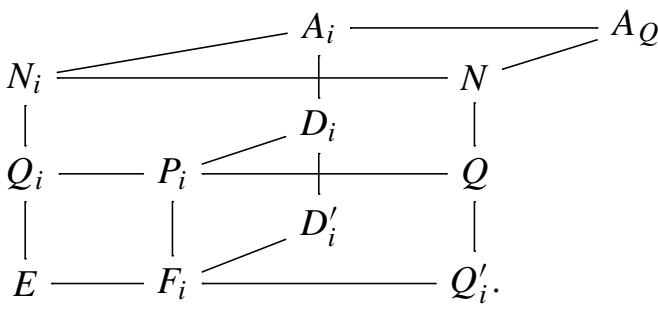

Let $A=\bigcap_{i \in I} A_{i}$. By Proposition 3.6, $A$ is a central simple $E$-algebra for which $A Q_{i}=A_{i}$ for each $i \in I$. In particular, $A=M_{k}(D)$ for some division algebra $D$ of index $n / k$.

Now, $D \otimes_{E} Q_{i}$ is Brauer equivalent to $A \otimes_{E} Q_{i} \cong A_{i}$, which is Brauer equivalent to $D_{i}$. Thus, $n / n_{i}=\operatorname{ind}\left(D_{i}\right) \mid$ ind $D$ for each $i \in I$ and $n=\operatorname{lcm}_{i}\left(n / n_{i}\right) \mid$ ind $D$. It follows that $k=1$ and $A$ is a division algebra. Naturally, $F$ is a subfield of $A$ and ind $A=[F: E]$. It follows that $F$ is a maximal subfield of the division algebra $A$.

By choosing a basis for $A / F$ and considering the corresponding structure constants one can form an $E$-division algebra $A^{\prime}$ which is $E$-isomorphic to $A$ such that $F^{\prime}$ is a maximal subfield of $A^{\prime}$.

We will show that $A^{\prime} \otimes_{E} E^{\prime}$ is an $E^{\prime}$-division algebra, but first let us show that this implies that $F^{\prime} E^{\prime} / E^{\prime}$ is an adequate $G$-extension (and hence $G$ is $E^{\prime}$-admissible). Indeed, if $A^{\prime} \otimes_{E} E^{\prime}$ is a division algebra, then $F^{\prime} \otimes_{E} E^{\prime}$ is a field. It follows that $F^{\prime} \otimes_{E} E^{\prime} \cong F^{\prime} E^{\prime}$, since $F^{\prime} \otimes_{E} E^{\prime}$ is $G$-Galois over $E^{\prime}$ [Saltman 1999, Theorem 6.3]. Thus, $\left[F^{\prime} E^{\prime}: E^{\prime}\right]=\left[F^{\prime}: E\right]$ and $F^{\prime} \cap E^{\prime}=E$. Since $F^{\prime} E^{\prime}$ splits $A^{\prime} \otimes_{E} E^{\prime}$ and as ind $\left(A^{\prime} \otimes_{E} E^{\prime}\right)=\left[F^{\prime} E^{\prime}: E^{\prime}\right], F^{\prime} E^{\prime}$ is a maximal subfield of $A^{\prime} \otimes_{E} E^{\prime}$ and hence an adequate $G$-extension.

In order to show that $A^{\prime} \otimes_{E} E^{\prime}$ is an $E^{\prime}$-division algebra, we first note that for each $i \in I, P_{i}=F_{i} Q_{i}=F^{\prime} Q_{i}$, by [Haran and Völklein 1996, Lemma 3.6(b)]. Thus, we have the diagram

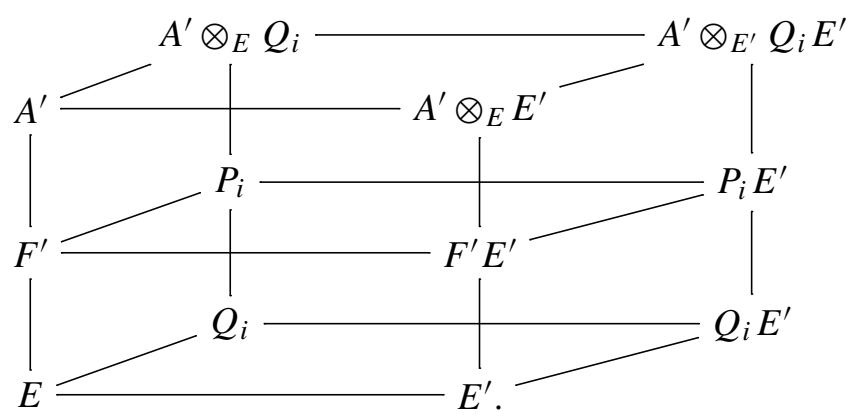

As mentioned above, $A^{\prime} \otimes_{E} Q_{i}$ is Brauer equivalent to $D_{i}=D_{i}^{\prime} \otimes_{E} Q_{i}$. Thus $A^{\prime} \otimes_{E} Q_{i} E^{\prime}$ is Brauer equivalent to $D_{i}^{\prime} \otimes_{Q_{i}} Q_{i} E^{\prime}$, which by the choice of $D_{i}^{\prime}$ is a 
division algebra. Then, for all $i \in I$,

$$
\frac{n}{n_{i}}=\operatorname{ind}\left(D_{i}^{\prime}\right)=\operatorname{ind}\left(D_{i}^{\prime} \otimes_{Q_{i}} Q_{i} E^{\prime}\right)\left|\operatorname{ind}\left(A^{\prime} \otimes_{E} Q_{i} E^{\prime}\right)\right| \operatorname{ind}\left(A^{\prime} \otimes_{E} E^{\prime}\right) .
$$

It follows that $n=\operatorname{lcm}_{i \in I}\left(n / n_{i}\right) \mid \operatorname{ind}\left(A^{\prime} \otimes_{E} E^{\prime}\right)$. Hence $n=\operatorname{ind}\left(A^{\prime} \otimes E^{\prime}\right)$, which shows that $A^{\prime} \otimes_{E} E^{\prime}$ is a division algebra.

As a corollary, we get our Main Theorem, which we restate for convenience:

Theorem 3.8. Let $R$ be an equicharacteristic complete local domain of dimension 2 , with a separably closed residue field. Let $E=$ Quot $R$ and let $G$ be a finite group of order not divisible by char $E$. Then $G$ is E-admissible if and only if all the Sylow subgroups of $G$ are abelian of rank at most 2.

Proof. By Proposition 3.7, if the Sylow subgroups of $G$ are abelian of rank at most 2 then $G$ is $E$-admissible. For the converse, assume $G$ is $E$-admissible. For a prime $v$ of $E$, let $\operatorname{ram}_{v}$ denote the ramification map $\operatorname{ram}_{v}: \operatorname{Br} E \rightarrow \mathrm{H}^{1}\left(G_{E_{v}}, Q / Z\right)$ [Saltman 1999]. Following [Harbater et al. 2009], we say that an $\alpha \in \operatorname{Br} E$ is determined by ramification with respect to a set of primes $\Omega$ if there is a prime $v \in \Omega$ for which $\exp (\alpha)=\exp \left(\operatorname{ram}_{v}(\alpha)\right)$. Let $D$ be an $E$-division algebra with maximal subfield $L$ that has Galois group $G=\operatorname{Gal}(L / E)$. Let $p=\operatorname{char} E$ (possibly $p=0$ ). By [Harbater et al. 2009, Theorem 3.3], if $D$ satisfies:

(1) the order of $D$ is prime to $p$ and ind $D=\exp D$, and

(2) $D$ is determined by ramification with respect to some set of discrete valuations whose residue characteristic is prime to $|G|$,

then $G$ has Sylow subgroups that are abelian of rank at most 2. Condition (1) is satisfied for any $\alpha$ of order prime to $p$ by Theorem 2.1 of [Colliot-Thélène et al. 2002], while condition (2) is satisfied by Corollary 1.9(c) of the same paper with respect to the set of codimension 1 primes of $R$.

Remark 3.9. Let $E$ be as above. By [Colliot-Thélène et al. 2002, Theorem 2.1], any Brauer class $\alpha \in \operatorname{Br} E$ of order prime to char $E$ has $\operatorname{ind}(\alpha)=\exp (\alpha)$. Thus by [Schacher 1968, Proposition 2.2], a subfield of an $E$-division algebra is also a maximal subfield of some $E$-division algebra.

\section{Acknowledgements}

The authors wish to thank David Harbater for useful comments, and the referee, for many comments and corrections that significantly improved the presentation of this work. 


\section{References}

[Bary-Soroker et al. 2008] L. Bary-Soroker, D. Haran, and D. Harbater, "Permanence criteria for semi-free profinite groups", preprint, 2008. To appear in Math. Ann. arXiv 0810.0845v2

[Colliot-Thélène et al. 2002] J.-L. Colliot-Thélène, M. Ojanguren, and R. Parimala, "Quadratic forms over fraction fields of two-dimensional Henselian rings and Brauer groups of related schemes", pp. 185-217 in Algebra, arithmetic and geometry (Mumbai, 2000), vol. 1, edited by R. Parimala, Tata Inst. Fund. Res. Stud. Math. 16, Tata Inst. Fund. Res., Bombay, 2002. MR 2004c: 14031 Zbl 1055.14019

[DeMeyer and Ingraham 1971] F. DeMeyer and E. Ingraham, Separable algebras over commutative rings, Lecture Notes in Math. 181, Springer, Berlin, 1971. MR 43 \#6199 Zbl 0215.36602

[Fein and Schacher 1995a] B. Fein and M. Schacher, " $\boldsymbol{Q}(t)$ and $\boldsymbol{Q}((t))$-admissibility of groups of odd order”, Proc. Amer. Math. Soc. 123:6 (1995), 1639-1645. MR 95g:12003 Zbl 0974.12003

[Fein and Schacher 1995b] B. Fein and M. Schacher, "Crossed products over algebraic function fields", J. Algebra 171:2 (1995), 531-540. MR 96b:12003 Zbl 0827.12005

[Fein et al. 1992] B. Fein, D. J. Saltman, and M. Schacher, "Brauer-Hilbertian fields", Trans. Amer. Math. Soc. 334:2 (1992), 915-928. MR 93b:12006 Zbl 0767.12003

[Feit 2004] W. Feit, "PSL 2 (11) is admissible for all number fields", pp. 295-299 in Algebra, arithmetic and geometry with applications (West Lafayette, IN, 2000), edited by C. Christensen et al., Springer, Berlin, 2004. MR 2004k:12006 Zbl 1121.12004

[Fresnel and van der Put 2004] J. Fresnel and M. van der Put, Rigid analytic geometry and its applications, Progress in Mathematics 218, Birkhäuser, Boston, MA, 2004. MR 2004i:14023 Zbl 1096.14014

[Fried and Jarden 2005] M. D. Fried and M. Jarden, Field arithmetic, 2nd ed., Ergebnisse der Math.

(3) 11, Springer, Berlin, 2005. MR 2005k:12003 Zbl 1055.12003

[Haran and Jarden 1998] D. Haran and M. Jarden, "Regular split embedding problems over complete valued fields", Forum Math. 10:3 (1998), 329-351. MR 99e:12007 Zbl 0903.12003

[Haran and Völklein 1996] D. Haran and H. Völklein, "Galois groups over complete valued fields", Israel J. Math. 93 (1996), 9-27. MR 97c:12002 Zbl 0869.12006

[Harbater 1987] D. Harbater, "Galois coverings of the arithmetic line", pp. 165-195 in Number theory (New York, 1984-1985), edited by D. V. Chudnovsky et al., Lecture Notes in Math. 1240, Springer, Berlin, 1987. MR 88h:14020 Zbl 0627.12015

[Harbater 2003] D. Harbater, "Patching and Galois theory", pp. 313-424 in Galois groups and fundamental groups, edited by L. Schneps, Math. Sci. Res. Inst. Publ. 41, Cambridge Univ. Press, 2003. MR 2004j:14030 Zbl 1071.14029

[Harbater and Hartmann 2007] D. Harbater and J. Hartmann, "Patching over fields", preprint, 2007. To appear in Israel Journal of Mathematics. arXiv 0710.1392v3

[Harbater and Stevenson 2005] D. Harbater and K. F. Stevenson, "Local Galois theory in dimension two", Adv. Math. 198:2 (2005), 623-653. MR 2007e:12002 Zbl 1104.12003

[Harbater et al. 2009] D. Harbater, J. Hartmann, and D. Krashen, "Patching subfields of division algebras", preprint, 2009. To appear in Transactions of the AMS. arXiv 0904.1594v2

[Jacobson 1996] N. Jacobson, Finite-dimensional division algebras over fields, Springer, Berlin, 1996. MR 98a:16024 Zbl 0874.16002

[Liedahl 1996] S. Liedahl, " $K$-admissibility of wreath products of cyclic p-groups", J. Number Theory 60:2 (1996), 211-232. MR 97j:11059 Zbl 0861.12002 
[Matsumura 1986] H. Matsumura, Commutative ring theory, Cambridge Studies in Advanced Math. 8, Cambridge University Press, 1986. MR 88h:13001 Zbl 0603.13001

[Neftin 2009] D. Neftin, "Admissibility and realizability over number fields", preprint, 2009. arXiv 0904.3772v1

[Paran 2008] E. Paran, "Algebraic patching over complete domains", Israel J. Math. 166 (2008), 185-219. MR 2009e:12006 Zbl 1159.12002

[Paran 2010] E. Paran, "Galois theory over complete local domains", preprint, 2010, Available at www.tau.ac.il/ paranela/LocalGal.pdf. To appear in Math. Annalen.

[Pierce 1982] R. S. Pierce, Associative algebras, Graduate Texts in Math. 88, Springer, New York, 1982. MR 84c:16001 Zbl 0497.16001

[Pop 1996] F. Pop, “Embedding problems over large fields", Ann. of Math. (2) 144:1 (1996), 1-34. MR 97h:12013 Zbl 0862.12003

[Pop 2010] F. Pop, "Henselian implies large”, preprint, 2010, Available at http://tinyurl.com/ 29dhoxl. To appear in Ann. Math.

[Reiner 1975] I. Reiner, Maximal orders, London Mathematical Society Monographs 5, Academic Press, London, 1975. MR 52 \#13910 Zbl 0305.16001

[Saltman 1999] D. J. Saltman, Lectures on division algebras, CBMS Regional Conference Series in Mathematics 94, Amer. Math. Soc., Providence, RI, 1999. MR 2000f:16023 Zbl 0934.16013

[Schacher 1968] M. M. Schacher, "Subfields of division rings, I", J. Algebra 9 (1968), 451-477. MR 37 \#2809 Zbl 0174.34103

[Schacher and Sonn 1992] M. Schacher and J. Sonn, " $K$-admissibility of $A_{6}$ and $A_{7}$ ", J. Algebra 145:2 (1992), 333-338. MR 93a:12003 Zbl 0739.12004

[Sonn 1983] J. Sonn, “ $Q$-admissibility of solvable groups”, J. Algebra 84:2 (1983), 411-419. MR 86b:20022 Zbl 0524.16007

[Stern 1982] L. Stern, Admissibility of finite groups over global fields, Ph.D. thesis, Technion, Haifa, 1982.

Communicated by Jean-Louis Colliot-Thélène

Received 2009-10-09 Revised 2010-02-15 Accepted 2010-03-21

neftind@tx.technion.ac.il Department of Mathematics,

Technion - Institute of Technology, Haifa 32000, Israel

paranela@post.tau.ac.il Einstein Institute of Mathematics, Edmond J. Safra Campus, Givat Ram, The Hebrew University of Jerusalem, Jerusalem 91904, Israel http://www.tau.ac.il/ paranela/ 


\section{Algebra \& Number Theory}

www.jant.org

\section{EDITORS}

\section{MANAGING EDITOR}

Bjorn Poonen

Massachusetts Institute of Technology

Cambridge, USA

\author{
EDITORIAL BOARD CHAIR \\ David Eisenbud \\ University of California \\ Berkeley, USA
}

\section{BOARD OF EDITORS}

\section{Georgia Benkart}

Dave Benson

Richard E. Borcherds

John H. Coates

J-L. Colliot-Thélène

Brian D. Conrad

Hélène Esnault

Hubert Flenner

Edward Frenkel

Andrew Granville

Joseph Gubeladze

Ehud Hrushovski

Craig Huneke

Mikhail Kapranov

Yujiro Kawamata

János Kollár

Hendrik W. Lenstra

Yuri Manin

Barry Mazur
University of Wisconsin, Madison, USA

University of Aberdeen, Scotland

University of California, Berkeley, USA

University of Cambridge, UK

CNRS, Université Paris-Sud, France

University of Michigan, USA

Universität Duisburg-Essen, Germany

Ruhr-Universität, Germany

University of California, Berkeley, USA

Université de Montréal, Canada

San Francisco State University, USA

Hebrew University, Israel

University of Kansas, USA

Yale University, USA

University of Tokyo, Japan

Princeton University, USA

Universiteit Leiden, The Netherlands

Northwestern University, USA

Harvard University, USA
Susan Montgomery

Shigefumi Mori

Andrei Okounkov

Raman Parimala

Victor Reiner

Karl Rubin

Peter Sarnak

Michael Singer

Ronald Solomon

Vasudevan Srinivas

J. Toby Stafford

Bernd Sturmfels

Richard Taylor

Ravi Vakil

Michel van den Bergh

Marie-France Vignéras

Kei-Ichi Watanabe

Andrei Zelevinsky

Efim Zelmanov
University of Southern California, USA

RIMS, Kyoto University, Japan

Princeton University, USA

Emory University, USA

University of Minnesota, USA

University of California, Irvine, USA

Princeton University, USA

North Carolina State University, USA

Ohio State University, USA

Tata Inst. of Fund. Research, India

University of Michigan, USA

University of California, Berkeley, USA

Harvard University, USA

Stanford University, USA

Hasselt University, Belgium

Université Paris VII, France

Nihon University, Japan

Northeastern University, USA

University of California, San Diego, USA

\section{PRODUCTION}

ant@mathscipub.org

Silvio Levy, Scientific Editor

Andrew Levy, Production Editor

See inside back cover or www.jant.org for submission instructions.

The subscription price for 2010 is US \$140/year for the electronic version, and \$200/year (+\$30 shipping outside the US) for print and electronic. Subscriptions, requests for back issues from the last three years and changes of subscribers address should be sent to Mathematical Sciences Publishers, Department of Mathematics, University of California, Berkeley, CA 94720-3840, USA.

Algebra \& Number Theory (ISSN 1937-0652) at Mathematical Sciences Publishers, Department of Mathematics, University of California, Berkeley, CA 94720-3840 is published continuously online. Periodical rate postage paid at Berkeley, CA 94704, and additional mailing offices.

ANT peer review and production are managed by EditFLOW ${ }^{\mathrm{TM}}$ from Mathematical Sciences Publishers.

\section{PUBLISHED BY}

mathematical sciences publishers

http://www.mathscipub.org

A NON-PROFIT CORPORATION

Typeset in LATEX

Copyright $\odot 2010$ by Mathematical Sciences Publishers 


\section{Algebra \& Number Theory}

Volume $4 \quad$ No. $6 \quad 2010$

Generalized moonshine I: Genus-zero functions

SCOTT CARNAHAN

Integral trace forms associated to cubic extensions

GUILLERMO MANTILLA-SOLER

RACHEL OLLIVIER

DANNY NEFTIN and ELAD PARAN

John E. Cremona, Tom A. Fisher and Michael Stoll 